\title{
STRUCTURAL VARIATIONS OF GRAPHANE
}

\author{
Tatiana E. Belenkova, Vladimir M. Chernov, Eugeny A. Belenkov \\ Chelyabinsk State University, http://www.csu.ru \\ 454001 Chelyabinsk, Russian Federation \\ belenkova_te@gmail.com, chernov@csu.ru, belenkov@csu.ru
}

Abstract. Calculations of the structure and electronic properties for five structural variations of graphane were performed within the framework of density functional theory (DFT) with generalized gradient approximations (DDA). The electron densities of states and band structure of graphene crystals have been calculated. It has been established that the band gap at the Fermi level for graphane polymorphs varies from $5.50 \mathrm{eV}$ to $5.65 \mathrm{eV}$. Energy sublimation graphane layers with different structure was varying from 11.33 to $11.48 \mathrm{eV} /$ atom.

Keywords: carbon, graphene, crystal structure, polymorphism, first principles calculations

PACS 81.05.ue

Bibliography - 11 references

Received 02.06.2016

RENSIT, 2016, 8(1):49-54

DOI: $10.17725 /$ rensit.2016.08.049

CONTENTS

1. Introduction (49)

2. Research Methodology (50)

3. Results and Discussion (50)

4. Conclusion (53)

REFERENCES (53)

\section{INTRODUCTION}

Graphene layers were first obtained in 2004 [1] in 2010 for their research was awarded the Nobel Prize in Physics. Use of graphene layers for the construction of electronic devices possible using cutting out of graphene layers ribbons having a lateral width of the order of a nanometer. Such graphene ribbons depending on the width and orientation of the carbon-carbon bonds can have conductive properties of the semiconductor to the metal, just as observed for carbon nanotubes. However, such tapes to sew in space with need for isolate them from each other is rather complicated technological problem. Much easier to obtain nanoelectronic devices from grapheneribbons forming them on graphane dielectric layers by removing part of the hydrogen atoms. Graphane layers are obtained by joining one hydrogen atom to each of the carbon atoms of the graphene layer. Such layers more properly be called hydrogenographane since they do consist not only of carbon atoms but also atoms of hydrogen. Theoretically, the possible existence of such compounds was predicted in 2007 [2], experimentally hydrogenographane layers were synthesized in 2009 [3]. Graphane (unlike graphene) is a dielectric. It is stable to hydrogen desorption at normal conditions, and therefore can be used in nanoelectronic devices operating at room temperature [4]. Hydrogenographane layers, both theoretically and experimentally investigated to date, consist of carbon atoms in the sp3 hybridization. Each of the carbon atoms linked by covalent $\sigma$-bonds to three neighboring carbon atoms in addition to each carbon atom of layer is joined by hydrogen atom alternately on each side of the layer $[2,3]$. Perhaps may exist structural types of hydrogenographane layers with different properties that may be of interest in terms of using them as a basis for electronic devices. In this study, we investigated the structure and electronic properties of the five main 
polymorphic types of hydrogenographane layers.

\section{RESEARCH METHODOLOGY}

For practical use of graphane layers it is desirable obtaining layers of graphane with non-carbon atoms attached to only one side of the layer. However, as showed studies carried out earlier $[5,6]$, these layers are strongly deformed and tend to form structure like a scroll, which makes them unsuitable for use as a basis for nanoelectronic devices. Therefore, in this study we analyzed only hydrogenographane layers with hydrogen atoms attached to both sides of graphane layer.

As shown by theoretical analysis - there are only five different ways in connection with non-carbon atoms graphane layer on both sides, so that the positions of all the carbon atoms were crystallographically equivalent. Various structural types of graphane layers with non-equivalent positions of carbon atoms may be more, but thermodynamically stable should be layers with equivalent positions of the atoms.

Fig. 1 shows the initial graphene layers with different dedicated color including the carbon atoms to which the non-carbon atoms can join with its upper-layer side (to the atoms of the same color), or the lower layer side (to atoms of a different color). The first of these types of connection T1 corresponds hydrogenographane layer described in $[2$, 3]. Three ways to join $\mathrm{T} 2, \mathrm{~T} 3$ and $\mathrm{T} 4$ were considered earlier in $[7,8]$. The possibility of existence the fifth main structural type T5 of hydrogenographane layers studied for the first time in this paper.

Theoretically built hydrogenographane layers of the five main types were subjected to geometrical optimization by the methods of density functional theory (DFT) [9] within the generalized gradient approximations (GGA) [10]. Calculations of geometrically optimized structure and band structure were made by software package Quantum ESPRESSO [11]. Calculations were carried out for the layers arranged in a three-dimensional structure, but the distance between the layers (at the same time it was a period of elementary broadcasts along the crystallographic c axis) was chosen sufficiently large $(10 \AA)$, so that layers do not affect the structure of the adjacent layers and their properties. Densities of electron states calculated using a set of k-points: $12 \times 12 \times 12$. The wave functions are displayed on a truncated basis set of plane waves. The dimension of the set of basis functions has been limited by the value $\mathrm{E}_{\text {cutoff }}=1 \mathrm{keV}$.

\section{RESULTS AND DISCUSSION}

Images five of structural types of graphane layers, geometrically optimized by DFTGGA, shown in Fig. 2. The structural characteristics of the layers are shown in Table 1. Hydrogenographane layers of various types have very different structure. The layers have different unit cells which differ parameters and number of contained atoms (Fig. 2, Tab. 1). The unit cells of graphane layer contains from 4 to 16 atoms and include crystalline hexagonal lattice (Hex), triclinic ( $\mathrm{Tr}$ ) or orthorhombic (Ort) syngonies. Density of hydrogenographane

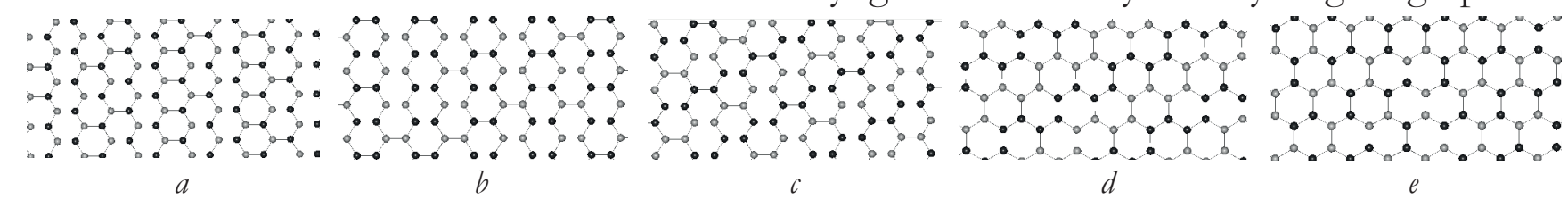

Fig. 1. Possible ways of attachment of bydrogen atoms on two sides of the graphene layer: (a) T1; (b) T2; (c) T3; (d) T4; (e) T5. 

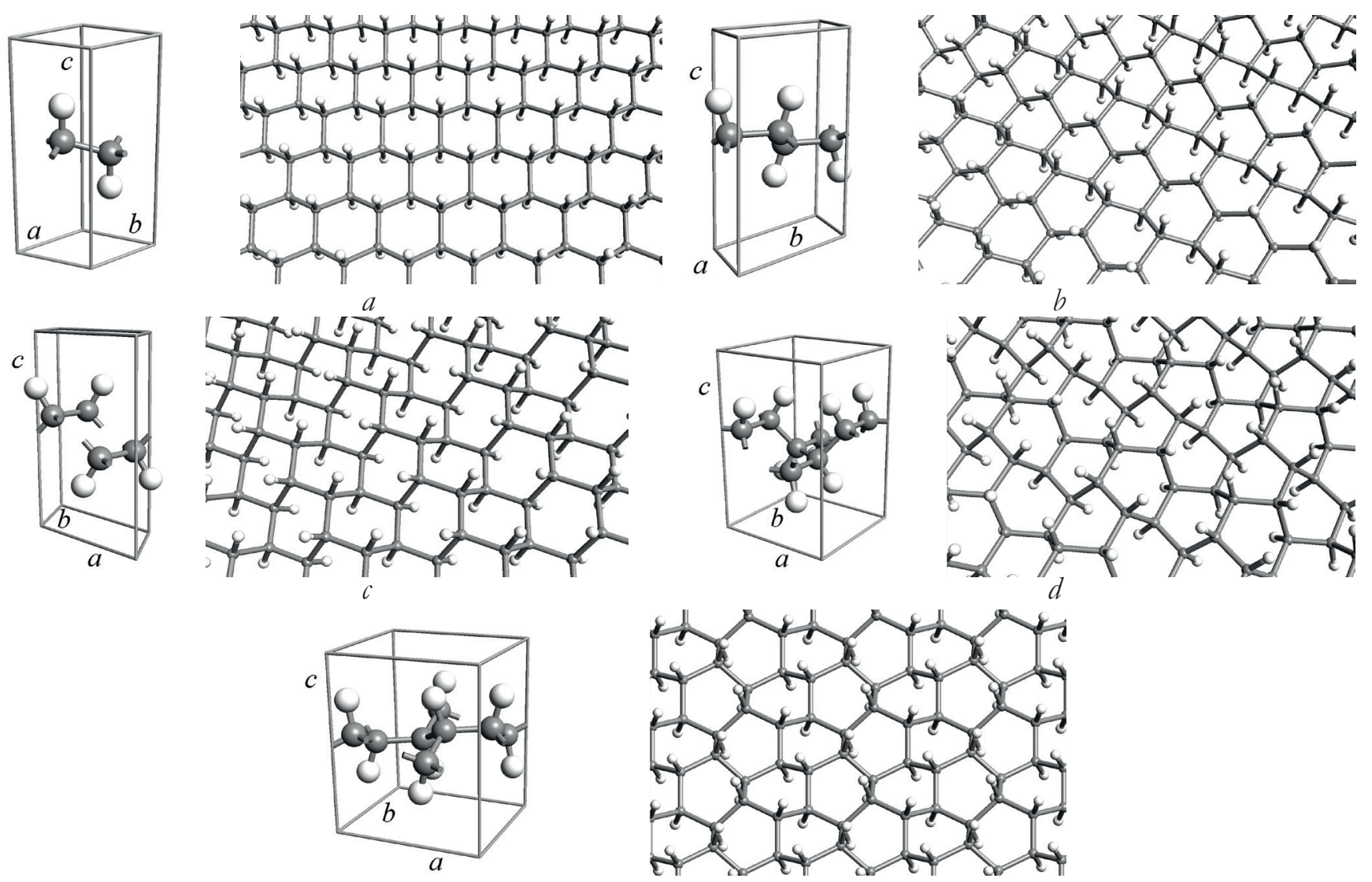

Fig. 2. Elementary cell structure and bydrogenographane layers of various types: (a) T1; (b) T2; (c) T3; (d) T4; (e) T5. layers ranges from 0.77 to $0.88 \mathrm{mg} / \mathrm{m}^{2}$ that total energy of a single C-H molecular group exceeds the density of graphene layers of in hydrogenographane layer and the energy $0.74 \mathrm{mg} / \mathrm{m}^{2}$. of isolated carbon and hydrogen atoms (the

The total energy per one C-H group of estimated value of these energies -149.56 and molecular (Etotal) ranges from -173.37 to $-12.33 \mathrm{eV} /$ atom.). In result of calculations -173.22 eV (Table 1). Sublimation energy were found specific sublimation energy per (Esub) calculation was made as a result of one carbon atom of graphane layer. The the calculation of the difference between the numerical values of sublimation energy

Table 1 The structural parameters and properties graphane layers T1-T5 types, as well as hexagonal graphene $\mathrm{L}_{6}$.

\begin{tabular}{|c|c|c|c|c|c|c|}
\hline Layer & T1 & $\mathrm{T} 2$ & T3 & $\mathrm{T} 4$ & T5 & $\mathrm{L}_{6}$ \\
\hline Syngony & Hex & $\mathrm{Tr}$ & $\mathrm{Tr}$ & Ort & Ort & Hex \\
\hline$a, \AA$ & \multirow[t]{2}{*}{2.5532} & 2.5415 & 4.6158 & 4.5707 & 5.0060 & \multirow[t]{2}{*}{2.491} \\
\hline$b, \AA$ & & 5.0214 & 2.5606 & 4.3496 & 4.4404 & \\
\hline $\mathrm{Y}^{\circ}{ }^{\circ}$ & 120 & 120.4393 & 123.7379 & 90 & 90 & 120 \\
\hline$E_{\text {total }}$, eV/u.c. & -346.74 & -693.08 & -693.29 & -1385.29 & -1385.74 & -314.64 \\
\hline$E_{\text {total }}, \mathrm{eV} /(\mathrm{CH})$ & -173.37 & -173.27 & -173.32 & -173.25 & -173.22 & - \\
\hline$E_{\text {sub }}$, eV/at. & 11.48 & 11.38 & 11.43 & 11.36 & 11.33 & 7.76 \\
\hline$\Delta E_{\text {sub }}$, eV/at. & 3.72 & 3.63 & 3.67 & 3.60 & 3.57 & 0 \\
\hline$\Delta, \mathrm{eV}$ & 5.55 & 5.50 & 5.54 & 5.51 & 5.65 & 0.00 \\
\hline$\rho, \mathrm{mg} / \mathrm{m}^{2}$ & 0.77 & 0.79 & 0.88 & 0.87 & 0.78 & 0.74 \\
\hline$N$, at. & 4 & 8 & 8 & 16 & 16 & 2 \\
\hline
\end{tabular}


hydrogenographane layers in the range of 11.33 to $11.48 \mathrm{eV} /$ atom that on $3.57 \div 3.72 \mathrm{eV}$ higher than the value of the specific energy of sublimation layer of hexagonal graphene (Table 1). Apparently this is due to higher strength carbon-hydrogen bond as compared with the carbon-carbon bond between the $\pi$ electrons of graphene layer. The maximum energy of sublimation is observed for the T1type layer, and the minimum for $\mathrm{T} 5$, but the relative energy difference is only $1.3 \%$, which indicates the possibility of stable existence of all main polymorphic types of graphane.

The results of measuring the lengths of interatomic bonds and the angles between them are shown in Table 2. The lengths of the carbon-carbon bonds vary in the range of 1.5459 do1.5830 $\AA$, and carbon-hydrogen from 1.1094 to $1.1183 \AA$. The angles between the bonds in graphane layers differ from the angle of $109.47^{\circ}$ that characteristic of the atomic positions in the diamond structure. The total deviation of angles between bonds from the angles in the diamond structure is characterized by a deformation parameter Def, which quantifies the degree of tension of the structure in comparison with the diamond structure. Minimum value Def $\left(10.87^{\circ}\right)$ was observed for graphane

Table 2

The bond lengths and bond angles in graphane layers.

\begin{tabular}{|c|c|c|c|c|c|}
\hline Layer & T1 & T2 & T3 & T4 & T5 \\
\hline $\mathrm{L}_{1(\mathrm{C}-\mathrm{C})}, \AA$ & 1.5467 & 1.5796 & 1.5530 & 1.5506 & 1.5701 \\
\hline $\mathrm{L}_{2(\mathrm{C}-\mathrm{C})}, \AA$ & 1.5467 & 1.5459 & 1.5530 & 1.5830 & 1.5476 \\
\hline $\mathrm{L}_{3(\mathrm{C}-\mathrm{C})}, \AA$ & 1.5467 & 1.5459 & 1.5530 & 1.5830 & 1.5559 \\
\hline $\mathrm{L}_{4(\mathrm{C}-\mathrm{H})}, \AA$ & 1.1183 & 1.1124 & 1.1128 & 1.1094 & 1.1139 \\
\hline$\varphi_{12^{\prime}},{ }^{\circ}$ & 111.25 & 112.27 & 112.28 & 112.44 & 112.00 \\
\hline$\varphi_{13^{\prime}}{ }^{\circ}$ & 111.25 & 112.27 & 112.28 & 112.44 & 117.77 \\
\hline$\varphi_{14},{ }^{\circ}$ & 107.63 & 106.61 & 107.85 & 107.42 & 105.94 \\
\hline$\varphi_{23^{\prime}},{ }^{\circ}$ & 111.25 & 110.60 & 111.04 & 112.39 & 107.44 \\
\hline$\varphi_{24},{ }^{\circ}$ & 107.63 & 107.41 & 106.50 & 106.47 & 106.55 \\
\hline$\varphi_{34},{ }^{\circ}$ & 107.63 & 107.41 & 106.50 & 106.47 & 106.45 \\
\hline Def, ${ }^{\circ}$ & 10.87 & 13.71 & 14.74 & 16.91 & 22.33 \\
\hline
\end{tabular}

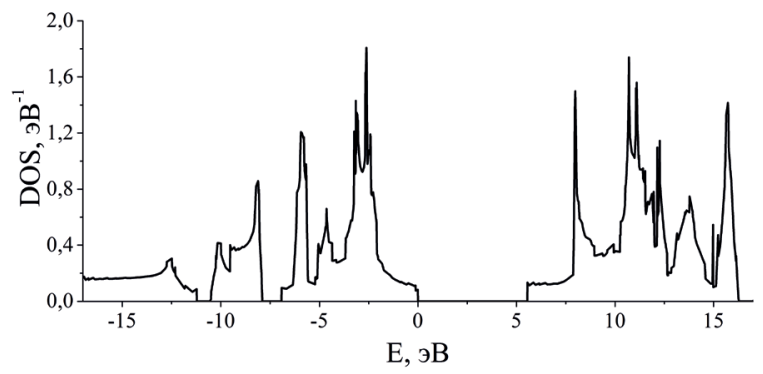

a

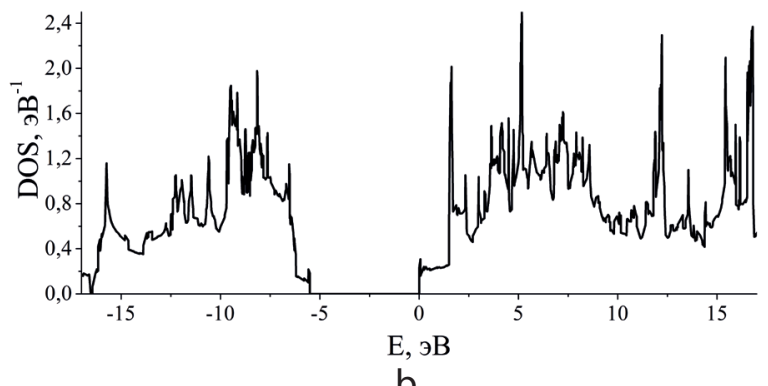

b

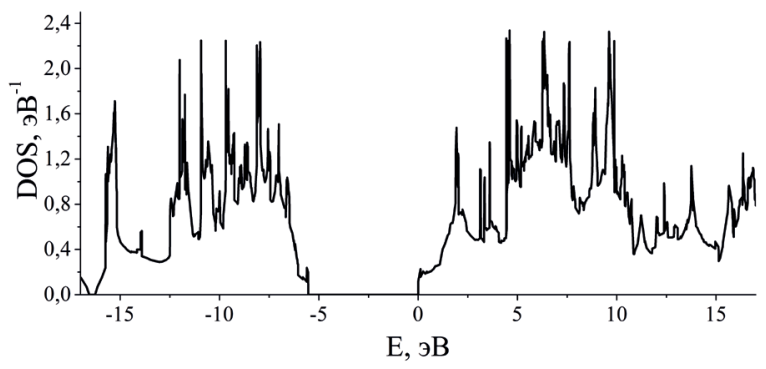

C

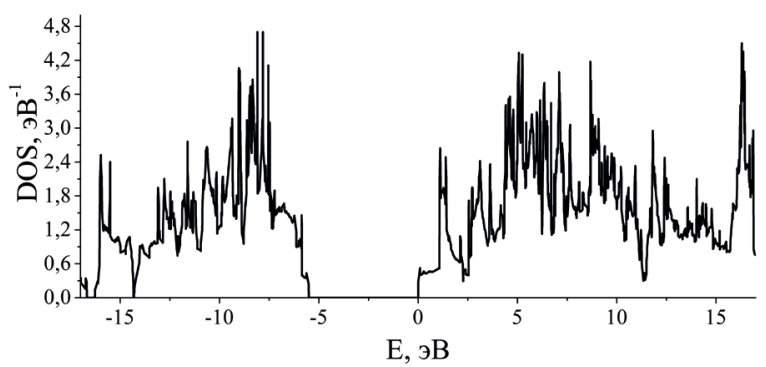

d

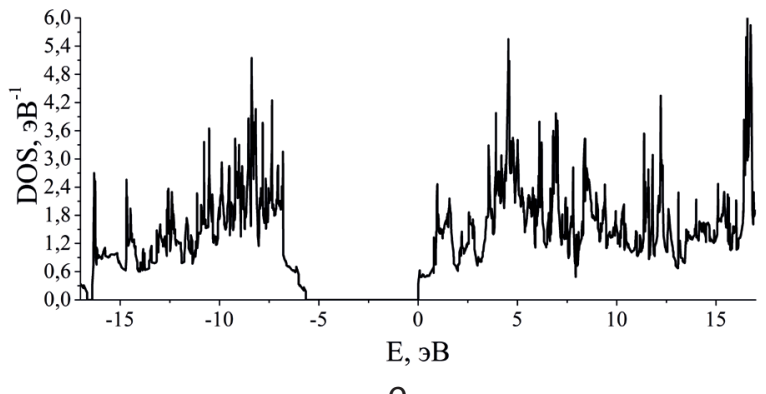

e

Fig. 3. The density of electron states hydrogenographane layers of different types: (a) T1; (b) T2; (c) T3; (d) T4; (e) T5.

T1-type layer, the maximum value $\left(22.33^{\circ}\right)$ for T5 layer. This is a good explanation of the difference sublimation energy structure types - a minimum of energy is observed 
for sublimation of the strained layer, the maximum $\mathrm{E}_{\text {sub }}$ - for the layer in which the smallest deformation parameter (Table 1,2.). The calculated of electron density of states for five graphane layers are shown in Fig. 3. The numerical values of band gaps at the Fermi energy level are shown in Table 1. The band gap $\Delta$ of graphene layers were in the range of 5.50 to $5.65 \mathrm{eV}$. This indicates that all hydrogenographane layers regardless of type should exhibit structural dielectric properties.

\section{CONCLUSION}

Thus, by method of DFT-GGA calculation were calculated the crystal structure and electronic properties of five main structural types of hydrogenographane layers, the resulting attachment of hydrogen atoms to the graphene layer $L_{6}$. Calculations of the band structure and density of electronic states graphyne compounds indicate that their electronic properties to be different from each other slightly, and all hydrogenographane layers should bad conduct electricity. Local carbonation and making of graphene ribbons on dielectric layer of graphane is possible for hydrogenographane layers of any of the five major structural types as a result can be obtained nanoelectronic devices. However, perhaps some of the polymorphic types of graphane may be preferable to deal with these problems. This question remains open and should be studied in future research.

\section{REFERENCES}

1. Novoselov KS, Geim AK, Morozov SV, Jiang D, Zhang Y, Dubonos SV, Grigorieva IV, Firsov AA. Electric field effect in atomically thin carbon films. Science, 2004, 306:666-669.
2. Sofo JO, Chaudhari AS, Barber GD. Graphane: A two-dimensional hydrocarbon. Phys. Rev. B, 2007, 75:153401.

3. Elias DC, Nair RR, Mohiuddin TM, Morozov SV, Blake P, Halsall MP, Ferrari AC, Boukhvalov DW, Katsnelson MI, Geim AK, Novoselov KS. Control of graphene's properties by reversible hydrogenation: evidence for graphane. Science, 2009, 323:610-613.

4. Openov LA, Podlivaev AI. Termicheskaya desirbtsiya vodoroda iz grafana [Thermal desorption of hydrogen from graphane]. Technical Physics Letters, 2010, 36(1):69-75.

5. Belenkova TE, Anfreeva AA. Modelirovanie struktury elektronnykh ustroystr na osnove selektivno grafitirovannykh grafanovykh sloev [Modeling the structure of electronic devices based on selectively carbonated graphane layers]. Theses VNKSF-18 (Krasnoyarsk, 2012). Ekaterinburg, ASF, 2012:102-103.

6. Belenkov EA, Shabiev FK. Scroll structure of carbon nanotubes obtained by the hydrothermal synthesis. Letters on materials, 2015, 5(4):459-462.

7. Wen XD, Hand L, Labet V, Yang T, Hoffmann R,1, Ashcroft NW, Oganov AR, Lyakhov AO. Graphane sheets and crystals under pressure. PNAS, 2011, 108(17):6833-6837.

8. Sorokin PB, Chernozatonskii LA. Poluprovodnikovye nanostruktury na osnove grafena [Graphene-based semiconductor nanostructures]. Phys. Usp., 2013, 56(2):105-122.

9. Koch WA, Holthausen MC. Chemist's Guide to Density Functional Theory. 2nd edition. Wiley-VCH Verlag GmbH, 2001, 293 p.

10. Perdew JP, Chevary JA, Vosko SH, Jackson KA, Pederson MR, Singh DJ, Fiolhais C. Atoms, molecules, solids, and 
surfaces: Applications of the generalized gradient approximation for exchange and correlation. Phys. Rev. B, 1992, 46:6671-6687.

11. Giannozzi P, Baroni S, Bonini N, Calandra M, Car R, Cavazzoni C, Ceresoli D, Chiarotti GL, Cococcioni M, Dabo I, Dal Corso A, Fabris S, Fratesi G, de Gironcoli S, Gebauer R, Gerstmann U, Gougoussis C, Kokalj A, Lazzeri M, Martin-Samos L, Marzari N, Mauri F, Mazzarello R, Paolini S, Pasquarello A, Paulatto L, Sbraccia C, Scandolo S, Sclauzero G, Seitsonen AP, Smogunov A, Umari P, Wentzcovitch RM. QUANTUM ESPRESSO: a modular and open-source software project for quantum simulations of materials. J. Phys.: Condens. Matter, 2009, 21:395502. 